\title{
On the time-optimal 2D-trajectories in non-uniform mediums
}

Citation for published version (APA):

Berger, A., Grigoriev, A., \& Usotskaya, N. (2011). On the time-optimal 2D-trajectories in non-uniform mediums. METEOR, Maastricht University School of Business and Economics. METEOR Research Memorandum No. 031 https://doi.org/10.26481/umamet.2011031

Document status and date:

Published: 01/01/2011

DOI:

10.26481/umamet.2011031

Document Version:

Publisher's PDF, also known as Version of record

\section{Please check the document version of this publication:}

- A submitted manuscript is the version of the article upon submission and before peer-review. There can be important differences between the submitted version and the official published version of record.

People interested in the research are advised to contact the author for the final version of the publication, or visit the DOI to the publisher's website.

- The final author version and the galley proof are versions of the publication after peer review.

- The final published version features the final layout of the paper including the volume, issue and page numbers.

Link to publication

\footnotetext{
General rights rights.

- You may freely distribute the URL identifying the publication in the public portal. please follow below link for the End User Agreement:

www.umlib.nl/taverne-license

Take down policy

If you believe that this document breaches copyright please contact us at:

repository@maastrichtuniversity.nl

providing details and we will investigate your claim.
}

Copyright and moral rights for the publications made accessible in the public portal are retained by the authors and/or other copyright owners and it is a condition of accessing publications that users recognise and abide by the legal requirements associated with these

- Users may download and print one copy of any publication from the public portal for the purpose of private study or research.

- You may not further distribute the material or use it for any profit-making activity or commercial gain

If the publication is distributed under the terms of Article $25 \mathrm{fa}$ of the Dutch Copyright Act, indicated by the "Taverne" license above, 


\section{Maastricht University}

Andre Berger, Alexander Grigoriev, Natalya Usotskaya

On the time-optimal 2Dtrajectories in non-uniform mediums

RM/11/031

\section{METEOR}

Maastricht University School of Business and Economics

Maastricht Research School of Economics

of Technology and Organization

\section{P.O. Box 616}

NL - 6200 MD Maastricht

The Netherlands 


\title{
On the Time-Optimal 2D-Trajectories in Non-Uniform Mediums
}

\author{
André Berger, Alexander Grigoriev, and Natalya Usotskaya \\ Department of Quantitative Economics, Maastricht University, \\ P.O. Box 616, 6200 MD Maastricht, The Netherlands \\ \{a.berger, a.grigoriev, n. usotskaya\}@maastrichtuniversity.nl
}

\begin{abstract}
This paper addresses two-dimensional trajectory optimization problems, where the mover's speed monotonically decreases/increases in one of the space's coordinates. For instance, it is well-known that the absolute value of the helicopter speed decreases in altitude because an air pressure drops. Then, it is not trivial to find a time-minimal helicopter trajectory from one given point to another.

We address such problems in different settings for the medium (atmosphere) and in the presence of obstacles. First, we consider the basic problem without any obstacles, where the mover's speed decreases linearly in altitude. We show that the problem is reducible to the L'Hopital's problem, one of the wellstudied problems in geometrical optics. In this case, the time-optimal trajectory is a circular segment, and therefore can be expressed in a closed analytic form.

Next, we address the problem with linear speed decrease in presence of rectilinear obstacles. We show that this problem can be solved in polynomial time. Finally, we consider the case without obstacles, where the medium is non-uniform and the mover's velocity is a piece-wise linear monotonically decreasing function. This is a widely accepted model in our motivating application of helicopter flights. In this case, we reduce the problem to the solution of a system of polynomial equations of fixed degree. Here, if the number of breakpoints of the velocity function is a constant, the algebraic elimination theory allows us to solve the problem in constant time with any given level of precision.
\end{abstract}

\section{Introduction}

The classical trajectory optimization problems in computational geometry usually assume a constant speed of the moving objects. Moreover, many fundamental geometric problems assume that a moving object, a mover, is a single point in two- or three-dimensional space. Under these two assumptions, length-optimal and time-optimal trajectories are the same. Therefore, dealing with a time-optimization problem, we can apply, for instance, the shortest-paths algorithms; see, e.g., $[1,2]$. The situation is totally different when we assume that the speed of the mover depends on the position in the space. In this case, length-optimal and time-optimal trajectories can deviate from each other significantly. One example for such a problem is finding shortest paths in weighted regions $[12,13]$, in which the plane is divided into a finite number of regions in each of which the speed is constant. In this paper we investigate such a shortest path problem where the speed changes continuously in one of the space's coordinates.

Given a mover in three-dimensional Euclidean space, assume that the absolute value of the mover's speed decreases or increases in one of the space coordinates. This is the real-life setting in any helicopter flight. The greater the altitude of the helicopter, the less the atmospheric pressure and, consequently, the less the air density. In turn, the reduction in air density will reduce the power available, and then, the maximum speed of the helicopter decreases with altitude. Though the (concave) function of the helicopter's maximum velocity in altitude is quite complex and hardly admits a closed analytical form, it is widely accepted to approximate it by linear or piece-wise linear functions with just few breakpoints, see, e.g. [3]. Therefore, we can formulate the basic helicopter problem as follows. Given is a source point $A$ and a destination point $B$ in three-dimensional Euclidean space, where, traditionally, the vertical $y$-axis represents points altitudes while the $x$ and $z$-axes represent the surface coordinates. No obstacles (also no ground level) are present. The 
absolute value of the mover's speed decreases linearly in altitude, i.e., $v(y)=\max \left\{v_{0}-q y, 0\right\}$, where $v_{0}>0$ is some speed intercept (for instance, the helicopter's maximum velocity at the ground level) and $q \geq 0$ is the velocity degradation rate. One has to find a time-optimal trajectory to fly the mover (helicopter) from $A$ to $B$. Notice that this three-dimensional problem can be easily reduced to the two-dimensional problem. This is because the time-optimal trajectory clearly belongs to the plane orthogonal to the surface and containing points $A$ and $B$. The general helicopter problem is the natural generalization of the basic helicopter problem, where the velocity is represented by the piece-wise linear function with finite number of breakpoints.

The helicopter problem with obstacles is formulated as follows: given points $A$ and $B$ in threedimensional Euclidean space, along with a set of polyhedral obstacles, find a time-optimal trajectory to.fly the helicopter from $A$ to $B$ without hitting the interior of any of the obstacles. It is noticeable that the helicopter problem with obstacles is a generalization of the classical three-dimensional Euclidean shortest-path problem: if all the velocity degradation rates are equal to zero, the problems are equivalent. It is well known that the two-dimensional Euclidean shortest-path problem is polynomially solvable [18], the three-dimensional problem is NP-hard [7], but admits polynomial time approximation schemes [1]. Notice that one can derive a polynomial-time approximation scheme to the three-dimensional general helicopter problem with obstacles by discretizing/scaling the space and constructing a weighted complete graph, where the edge weight is the time to travel between two vertices using the straight line trajectory. The presence of obstacles can be taken into account setting the edge weight to positive infinity if the straight line between two vertices hits interior of an obstacle. Now we can search for the shortest path in the obtained graph.

In the time-optimization setting, to tackle the general problem with obstacles, one might need a complete characterization of the set of optimal solutions to the basic problem without obstacles, the set of, so-called, motion primitives. A typical illustration of lifting from motion primitives to solutions to the general problem can be found, for example, in robotics, see [8-10]. In this paper we derive motion primitives for the basic helicopter problem. We show that the basic helicopter problem is reducible to the L'Hopital's problem [4], one of the most studied problems in geometrical optics and mechanics. As a consequence we have that the trajectory following a certain circle segment with endpoints $A$ and $B$ is the time-optimal trajectory in the class of continuously differentiable functions. We use Euler-Lagrange equations from the calculus of variations. One can see this also as a variant of the Pontryagin Maximum (Minimum) Principle [20]. This type of techniques is quite common in optimal control theory in general, and in robotics in particular; see, e.g., $[5,6]$.

Then, we generalize results for the basic helicopter problem in two ways. First, we show that the basic helicopter problem with rectilinear obstacles is solvable in polynomial time by a dynamic programming algorithm. Second, we reduce the general helicopter problem without obstacles to the system of fixed degree multivariable polynomial equations. If the number of breakpoints in the piece-wise linear velocity function is a constant, the resulting system has a fixed number of variables and it can be solved in polynomial time by the algebraic elimination theory; see, e.g., [11].

\section{Solution to the basic helicopter problem}

First, we notice that if the points $A=\left(x_{1}, y_{1}\right)$ and $B=\left(x_{2}, y_{2}\right)$ lay on the same vertical line (i.e., they share the same $x$-coordinate: $x_{1}=x_{2}$ ), then the time-optimal trajectory $L_{A B}$ is just a piece of the straight line $x=x_{1}$ between $A$ and $B$. We omit the proof as it is straightforward. We also assume that $q>0$, for otherwise we are in the well-studied setting of the classical Euclidean shortest-path problem. Thus, from now on we consider only the case where $x_{1} \neq x_{2}$ and $q>0$. We show that in this case the circle segment is the unique time-optimal trajectory. The first observation is about the convexity of any time-optimal trajectory. 
Lemma 1. For any two points $A$ and $B$ in $\mathbb{R}^{2} \cap\left\{(x, y): v_{0}-q y>0\right\}$, a time-optimal trajectory between $A$ and $B$ is a convex function of $x$.

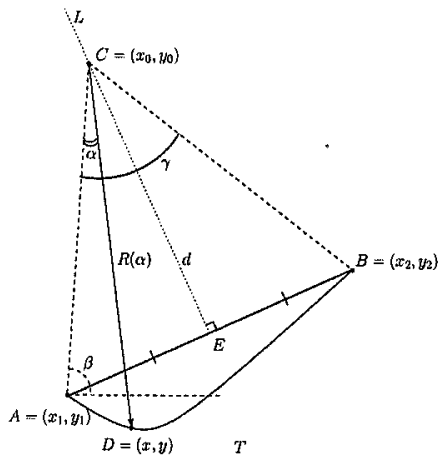

Fig. 1. Convex trajectory $T$ in the polar system of coordinates

By Lemma 1, we can restrict our search for time-optimal trajectories to the class of convex functions in $x$. Let $T$ be an arbitrary continuously differentiable convex trajectory between $A$ and $B$. Let $L$ be the line equidistant from $A$ and $B$. Since $T$ is convex in $x$, it is possible to observe every point of $T$ from any point $C$ on $L$ that lies above the line segment $A B$. We consider the trajectory $T$ in the polar system of coordinates with observation point $C=\left(x_{0}, y_{0}\right)$, where $C \in L$ is chosen such that the velocity in $C$ is 0 , i.e., $y_{0}=\frac{v_{0}}{q}$. For illustration see Figure 1 . We refer to the angle between $C A$ and the $\mathrm{x}$-axis as $\beta$ and to the angle between $C A$ and $C B$ as $\gamma$. Consider an arbitrary point $D=(x, y)$ of the trajectory $T$. In the chosen polar system of coordinates, the point $D$ is completely determined by $\alpha$ and $R(\alpha)$, where $\alpha$ is the angle between $C A$ and $C D$, and $R(\alpha)$ is the length of the interval $C D$ :

$$
\begin{aligned}
& x=x_{0}-R(\alpha) \cos (\alpha+\beta), \\
& y=y_{0}-R(\alpha) \sin (\alpha+\beta) .
\end{aligned}
$$

First, we write the integral which represents the time needed to travel along $T$. For a sufficiently small piece of the trajectory, we may assume that the velocity $v$ remains constant within the piece. Let the length of the piece be denoted by $\Delta l$, then the time to travel along the piece is $\Delta t \approx \frac{\Delta l}{v}$. The velocity $v$ is completely determined by the altitude of $D=(x, y)$. Therefore, by Equation (2), we have $v=v_{0}-q y=q R(\alpha) \sin (\alpha+\beta)$, as $v_{0}-q y_{0}=0$ by the choice of $C$. We know that for continuously differentiable trajectories the length of the piece $\Delta l$ is determined by $\Delta l=\sqrt{R^{\prime 2}(\alpha)+R^{2}(\alpha)} \Delta \alpha$, see, e.g. [16]. Therefore, the time needed to travel along $T$ is determined by the following integral:

$$
t_{T}=\int_{0}^{\gamma} \frac{\sqrt{R^{\prime 2}(\alpha)+R^{2}(\alpha)} d \alpha}{q R(\alpha) \sin (\alpha+\beta)}=\frac{1}{q} \int_{0}^{\gamma} \sqrt{\left(\frac{R^{\prime}(\alpha)}{R(\alpha)}\right)^{2}+1} \frac{d \alpha}{\sin (\alpha+\beta)} .
$$

Equation (3) allows us to proof the following theorem about the unique time-optimal trajectory between $A$ and $B$. We defer the proof to the journal version of the paper, see also Appendix A.

Theorem 1. Let $C$ be the intersection point of the line $y=\frac{v_{0}}{q}$ and the line equidistant from $A$ and $B$. The segment $T_{A B}$ of the circle with center $C$ and radius $R=|C A|=|C B|$ is a time-optimal trajectory between $A$ and $B$. The time needed to travel along $T_{A B}$ is

$$
t_{\text {opt }}=t_{T_{A B}}=\frac{1}{q} \int_{0}^{\gamma} \frac{d \alpha}{\sin (\alpha+\beta)}=\frac{1}{q} \ln \left|\frac{\tan \frac{\beta}{2}+\tan \frac{\gamma}{2}}{\tan \frac{\beta}{2}-\tan ^{2} \frac{\beta}{2} \tan \frac{\gamma}{2}}\right| .
$$


Here, $\tan \frac{\gamma}{2}$ and $\tan \frac{\beta}{2}$ can be determined in terms of coordinates $A=\left(x_{1}, y_{1}\right)$ and $B=\left(x_{2}, y_{2}\right)$. Point $C=\left(x_{0}, y_{0}\right)$ has an altitude $y_{0}=\frac{v_{0}}{q}$. Since $C$ belongs to the line $L$ equidistant from $A$ and $B$, we have following set of values:

$$
\begin{aligned}
x_{0} & =-\frac{y_{2}-y_{1}}{x_{2}-x_{1}} y_{0}-\frac{x_{1}^{2}+y_{1}^{2}-x_{2}^{2}-y_{2}^{2}}{2\left(x_{2}-x_{1}\right)}, \\
R & =|C A|=\sqrt{\left(x_{0}-x_{1}\right)^{2}+\left(y_{0}-y_{1}\right)^{2}}=\sqrt{\left(x_{0}-x_{2}\right)^{2}+\left(y_{0}-y_{2}\right)^{2}},
\end{aligned}
$$

Now we determine the tangents of $\beta$ and $\gamma$ in the following way: $\tan \frac{\gamma}{2}=\frac{\sqrt{R^{2}-d^{2}}}{d}$. Since $\sin \beta=\frac{y_{0}-y_{1}}{R}$ and $\cos \beta=\frac{x_{0}-x_{1}}{R}$, then $\tan \frac{\beta}{2}=\frac{\sin \beta}{1+\cos \beta}=\frac{y_{0}-y_{1}}{R+x_{0}-x_{1}}$, where $d=\sqrt{\left(x_{0}-\frac{x_{1}+x_{2}}{2}\right)^{2}+\left(y_{0}-\frac{y_{1}+y_{2}}{2}\right)^{2}}$.

To demonstrate the reducibility of the problem to the L'Hopital's problem, we present the time needed to travel along the trajectory $T$ in the following way:

$$
t_{T}=\int_{y_{1}}^{y_{2}} \frac{\sqrt{1+\dot{x}^{2}(y)}}{v_{0}-q y} d y
$$

as we have to go from the altitude $y_{1}$ to the altitude $y_{2}$ along the trajectory $x(y)$, when speed depends linearly at altitude, so $v(y)=v_{0}-q y$, and the length of the trajectory piece is $\Delta l=\sqrt{1+\dot{x}^{2}(y)}$, see [4]. This integral can be seen as a solution to the L'Hopital's problem, see [4], Chapter 3.

\section{The basic helicopter problem with rectilinear obstacles}

In this section we consider the case with rectilinear obstacles in 2D. The obstacles are given as the sequence of points $S=\left\{\left(x_{1}, y_{1}\right), \ldots,\left(x_{n}, y_{n}\right)\right\}$, so that every pair of consecutive points have exactly one same coordinate (either $x_{i}$ or $y_{i}$ ) and these same coordinates alternate, see Figure 2 . We assume that the helicopter goes from the left to the right without any loops, furthermore, the start point $A=\left(x_{1}, y_{1}\right)$ and the end point $B=\left(x_{2}, y_{2}\right)$ belong to the set $S$, otherwise we "draw" an additional step on the left of the terrain with the corner $A$, for example, and add $A$ to $S$. Here and below we refer to the straight line segment between any two points $K$ and $M$ as $L_{K M}$ and to the time-optimal circle segment between $K$ and $M$ from Theorem 1 as $T_{K M}$, the center of this circle is $C_{K M}=\left(x_{0}, y_{0}\right)$, where $y_{0}=\frac{V_{0}}{q}$ and $x_{0}$ and radius $R_{K M}$ are determined by Equations (5)-(6) respectively.

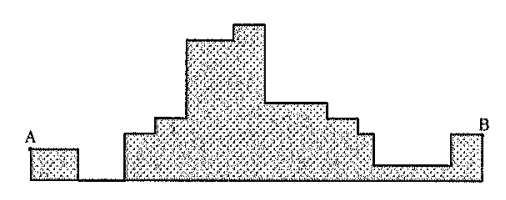

Fig. 2. The helicopter problem with rectilinear obstacles

\subsection{Dynamic programming algorithm}

The idea for the algorithm computing a time-optimal two-dimensional helicopter trajectory in presence of rectilinear obstacles is to find all potential breakpoints such that the trajectory changes the motion primitives in these points. If the amount of these points is polynomial then a simple 
dynamic program can solve the problem in polynomial time. Obviously, the points of the set $S$ are already potential breakpoints. Are there any other points? The answer is "yes" and it is given in the following lemma. It considers the time-optimal trajectory with respect to the one step obstacle, see Figure 3. The proof is also deferred to the journal version of the paper, see also Appendix B.

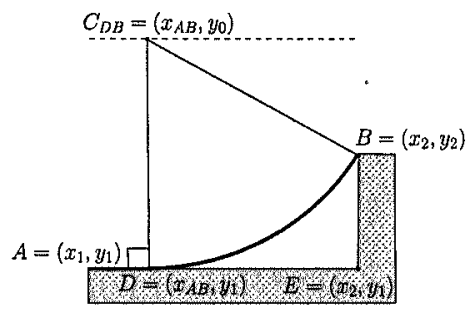

Fig. 3. The optimal trajectory for the one step case, $T_{A B}$ hits $L_{A E}$

Lemma 2. For three consecutive points $A=\left(x_{1}, y_{1}\right), E=\left(x_{2}, y_{1}\right)$ and $B=\left(x_{2}, y_{2}\right)$ from $S$, such that $y_{1}<y_{2}$, the time-optimal trajectory from $A$ to $B$ with obstacles is defined in the following way:

a) if $C_{A B}$ is to the left of $A$ then $T_{A B}$ is a time-optimal trajectory,

b) if $C_{A B}$ is to the right of $A$ then time-optimal trajectory is the combination of the straight horizontal line segment $L_{A D}$ and the circle segment $T_{D B}$, where $D$ and $C_{D B}$ determine a vertical line. In particular,

$$
D=\left(x_{2}-\sqrt{\left(y_{2}-y_{1}\right)\left(2 y_{0}-y_{1}-y_{2}\right)}, y_{1}\right) .
$$

We observe that by Lemma 2 for any pair of points $A=\left(x_{1}, y_{1}\right), B=\left(x_{2}, y_{2}\right)$ from $S$, it is sufficient to introduce at most one potential breakpoint $D=\left(x_{A B}, y_{1}\right)$ on the horizontal line $y=y_{1}$, if it is impossible to go along $T_{A B}$ and it is possible to go along the straight line $L_{A D}$ and circle segment $T_{D B}$. If there are other obstacles between $A$ and $B$ and the new trajectory hits some obstacle, then the time-optimal trajectory with rectilinear obstacles from $A$ to $B$ passes some other potential breakpoint $E$ between them. This fact is stated in Lemma 3. Hence, all necessary potential breakpoints are determined by Lemma 2 . The main ingredients to prove Lemma 3 are the following observations, the proofs are sketched in Appendix C:

Observation 1 For any three points $A=\left(x_{1}, y_{1}\right), B=\left(x_{2}, y_{2}\right)$ and $M=\left(x_{3}, y_{3}\right)$ such that $x_{1}<$ $x_{3}<x_{2}$ and $y_{3}<y_{1}, y_{3}<y_{2}, T_{A B}$ lies above $T_{A M}$ and $T_{M B}$ on the interval $\left[x_{1}, x_{2}\right]$.

Observation 2 For any three consecutive points $A=\left(x_{1}, y_{1}\right), C=\left(x_{2}, y_{1}\right)$ and $B=\left(x_{2}, y_{2}\right)$ such that $x_{1}<x_{2}$ and $T_{A B}$ hits $L_{A C}$, the time-optimal trajectory lies above $T_{A B}$ on the interval $\left[x_{1}, x_{2}\right]$.

We conclude the set of observations with the so-called "ground level case", when the unique obstacle between $A$ and $B$ is a horizontal line.

Observation 3 Given four consecutive points $A=\left(x_{1}, y_{1}\right), C=\left(x_{1}, y_{2}\right), D=\left(x_{2}, y_{2}\right)$ and $B=$ $\left(x_{2}, y_{3}\right)$ from $S$ such that $x_{1}<x_{2}, y_{2}<y_{1}, y_{2}<y_{3}$ and $T_{A B}$ hits $L_{C D}$, the time-optimal trajectory with obstacles is the combination of the circle segment $T_{A K}$, straight line $L_{K L}$ and circle segment $T_{L B}$, where $K$ and $L$ are the points of intersection of $T_{A D}$ and $T_{C B}$ with $L_{C D}$ respectively.

Finally, we present Lemma 3 . The proof is deferred to the journal version of the paper, see also Appendix C. It is the extensive case study for the obstacle patterns between two points $A$ and $B$. 
Lemma 3. If the time-optimal trajectory $T_{A B}$ between any two potential breakpoints $A=\left(x_{1}, y_{1}\right), B=$ $\left(x_{2}, y_{2}\right), x_{1}<x_{2}$, hits the obstacles, then there exists a potential breakpoint $C$ between $A$ and $B$ such that the time-optimal trajectory with obstacles from $A$ to $B$ passes $C$.

Now the problem is reduced to the problem of finding a shortest path in a weighted graph. All the potential breakpoints are the vertices of the graph, the number of vertices is not more than $n+n^{2}=O\left(n^{2}\right)$. There is an edge between two vertices if the time-optimal trajectory between corresponding potential breakpoints, given by Lemma 2, does not hit any obstacle. The time needed to go along this trajectory denotes the weight of the edge. Lemma 3 guarantees that the graph is connected and the time-optimal trajectory corresponds to the shortest path in the weighted graph. This problem is solvable in time $O\left(|V|^{2}\right)$, see, e.g. [19]. Hence, we have $O\left(n^{4}\right)$-time algorithm for 2D basic helicopter problem with rectilinear obstacles.

\section{General helicopter problem}

In this section we consider the general helicopter problem where the function of velocity is a piecewise linear concave function in altitude with finite number of breakpoints $y_{1}, \ldots, y_{n}$, see Figure 4 . Let $V_{i}, i \in I$ denote the velocity at the altitude $y_{i}$. By concavity of the function we have that $q_{1} \leq q_{2} \leq \ldots \leq q_{n+1}$. This is a reasonable assumption as the helicopter looses the speed quicker on high altitudes. Let us define the altitude layers $L_{i}, i \in I=\{0, \ldots n\}$, in the following natural way: $L_{i}=\left\{y \mid y_{i} \leq y \leq y_{i+1}\right\}, i \in I \backslash\{0, n\}, L_{0}=\left\{y \mid y \leq y_{1}\right\}$ and $L_{n}=\left\{y \mid y \geq y_{n}\right\}$. Therefore, the velocity function is given as follows:

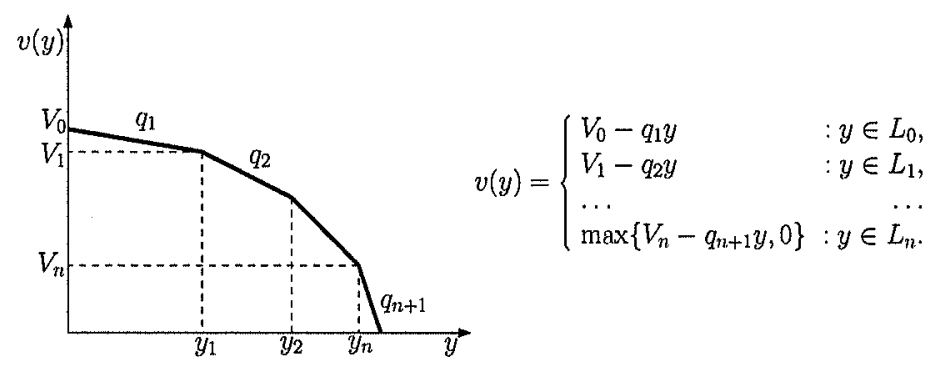

Fig. 4. Piece-wise linear velocity function

Consider the general helicopter problem where the helicopter must fly from $A=\left(x_{A}, y_{0}\right)$ to $B=\left(x_{B}, y_{n+1}\right)$. Without loss of generality assume, that $x_{A}<x_{B}$ and $y_{0} \in L_{0}, y_{n+1} \in L_{n}$. This setting yields several natural restrictions. First, we observe that the time-optimal trajectory from $A$ to $B$ is the set of circle and linear segments, as within one layer $L_{i}, i \in\{0, \ldots, n\}$, it is either the circle segment or the linear segment, see Section 2. Second, for any $y \geq y_{1}$ the timeoptimal trajectory is the monotonically increasing function. The reason is as follows. Assume that a non-increasing trajectory $T_{K B}$ is determined as the time-optimal trajectory within the layer $L_{1}$, see Figure 5. Then the piece $T_{K L}$ of this trajectory, which belongs to the layer $L_{0}$, is not optimal anymore. It is better to follow $T_{A L}$ within the layer $L_{0}$ and then continue with $T_{L B}$, a contradiction.

As the time-optimal trajectory is the set of circle and straight line segments, we restrict ourselves first to the case when only circle segments are allowed. The reason is to make the proofs less complicated. Later on we show how to generalize the result to the case of linear and circle segments. 


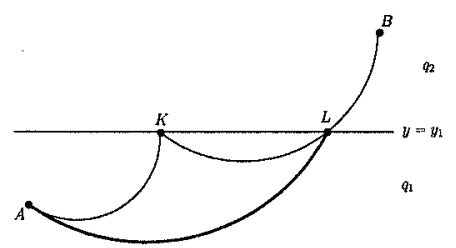

Fig. 5. Monotonically increasing circle segments when $y \geq y_{1}$. The trajectory $T_{A L}$ plus $T_{L B}$ is better than $T_{A K}$ plus $T_{K B}$

\subsection{Only circle segments}

In this subsection we consider the setting when all the pieces of the time-optimal trajectory from $A$ to $B$ are circle segments, hence the time needed to go along this trajectory can be calculated as the sum of times to go along the circle segments in layers $L_{i}$ 's, $i \in\{0, \ldots, n\}$.

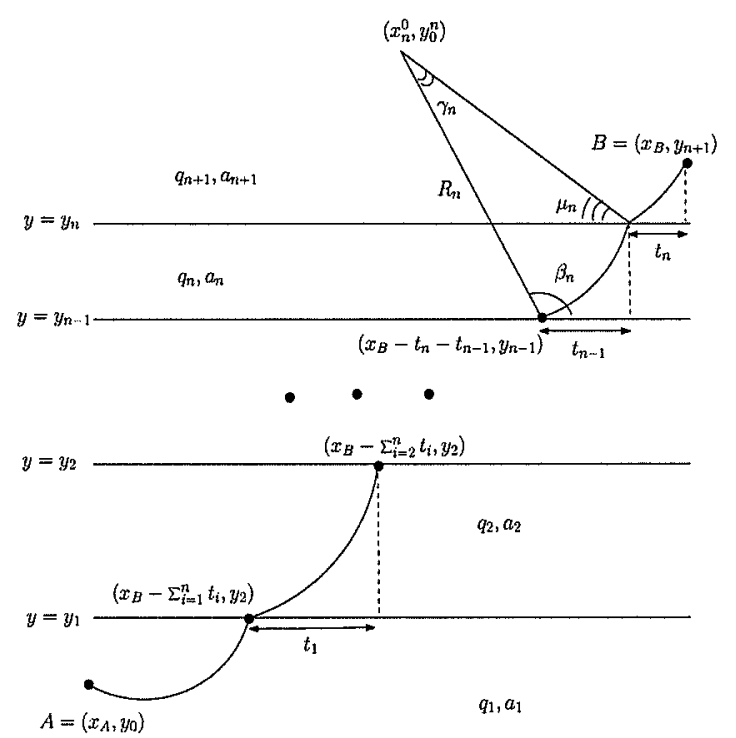

Fig. 6. Time-optimal trajectory is the set of circle segments, $x_{0}^{i}, \beta_{i}, \mu_{i}, \gamma_{i}$ and $R_{i}$ are functions at $t_{1}, \ldots, t_{n}$

For every layer $L_{i}, i \in\{0, \ldots, n\}$ we introduce the set of parameters, see Figure 6:

$-y_{0}^{i+1}=\frac{V_{i}}{q_{i+1}}$ is the altitude of the time-optimal circle segment center on this layer,

$-a_{i+1}=\sqrt{\left(2 y_{0}^{i+1}-y_{i+1}-y_{i}\right)\left(y_{i+1}-y_{i}\right)}$, as determined in Lemma 2. It is the maximal horizontal distance between two points on lines $y=y_{i}$ and $y=y_{i+1}$ such that the time-optimal circle segment is a monotonically increasing function,

and a set of variables and functions:

$-t_{i}$, such that for any $i$ greater than zero $t_{i}$ is the horizontal distance between the points of intersection the lines $y=y_{i}$ and $y=y_{i+1}$ by the time-optimal trajectory from $A$ to $B$, so that the coordinates of intersection are $\left(x_{B}-\sum_{i=k}^{n} t_{i}, y_{k}\right), k \in\{1, \ldots, n\}$. The values of the $t_{i}$ 's 
are strictly positive, as otherwise we have the vertical line segment, and $t_{i} \leq a_{i+1}, i>0$, as the circle segment should be a monotonically increasing function for $i>0$. We introduce the redundant variable $t_{0}: x_{B}-\sum_{i=0}^{n} t_{i}=x_{A}$, where $t_{0}$ is the horizontal distance between point $A$ and the point of intersection of the time-optimal trajectory from $A$ to $B$ with the line $y=y_{1}$. It is strictly positive as otherwise we go further to the left than point $A$, which is not optimal. It can be bigger than $a_{1}$ as within the layer $L_{0}$ the time-optimal trajectory is not necessary the monotonically increasing function,

$-x_{0}^{i+1}\left(t_{1}, \ldots, t_{n}\right)$ is the $x$-coordinate of the time-optimal circle segment center on this layer,

$-R_{i+1}\left(t_{1}, \ldots, t_{n}\right)$ is the radius of the time-optimal circle segment on this layer,

$-\beta_{i+1}\left(t_{1}, \ldots, t_{n}\right)$ and $\gamma_{i+1}\left(t_{1}, \ldots, t_{n}\right)$ are the angles defined in Theorem 1 , and $\mu_{i+1}\left(t_{1}, \ldots, t_{n}\right)$ is the angle between $C B$ and $x$-axis in terms of Figure 1, in particular, $\mu_{i+1}=\pi-\gamma_{i+1}-\beta_{i+1}$.

Now, we can write down the time needed to go alone this trajectory as the sum of the times to go alone the time-optimal trajectories within one layer $L_{i}$, see Section 2:

$$
t\left(t_{1}, \ldots, t_{n}\right)=\sum_{k=0}^{n} \frac{1}{q_{k+1}} \int_{0}^{\gamma_{k+1}\left(t_{1}, \ldots, t_{n}\right)} \frac{d \alpha}{\sin \left(\alpha+\beta_{k+1}\left(t_{1}, \ldots, t_{n}\right)\right)} .
$$

To minimize $t\left(t_{1}, \ldots, t_{n}\right)$, let us first find the stationary points of the function, so we have to find the partial derivatives of $t\left(t_{1}, \ldots, t_{n}\right)$ with respect to $t_{i}, i \in\{1, \ldots, n\}$ and equate them with zero. Direct differentiation leads to the following results, see Appendix $B$ for the details:

$$
\frac{\partial t\left(t_{1}, \ldots, t_{n}\right)}{\partial t_{i}}=-\sum_{k=1}^{n+1}\left[\frac{\partial \beta_{k}\left(t_{1}, \ldots, t_{n}\right)}{\partial t_{i}} \frac{1}{\sin \beta_{k}\left(t_{1}, \ldots, t_{n}\right)}+\frac{\partial \mu_{k}\left(t_{1}, \ldots, t_{n}\right)}{\partial t_{i}} \frac{1}{\sin \mu_{k}\left(t_{1}, \ldots, t_{n}\right)}\right]=0 .
$$

We find $\beta_{k}\left(t_{1}, \ldots, t_{n}\right)$ and $\mu_{k}\left(t_{1}, \ldots, t_{n}\right)$, for $k \geq 2$, using the formulas from Section 2 , as all the derivatives in this case can be obtained in the similar way. The case $k=1$ differs in the number of variables and it is considered later. Within the layer $L_{k}, k \geq 2$, the time-optimal trajectory goes from the point $\left(x_{B}-\sum_{i=k-1}^{n} t_{i}, y_{k-1}\right)$ to the point $\left(x_{B}-\sum_{i=k}^{n} t_{i}, y_{k}\right)$, so from the Equations (5)-(6) and the following formulae for the trigonometrical functions:

$$
\begin{aligned}
x_{0}^{k}\left(t_{1}, \ldots, t_{n}\right) & =-\frac{a_{k}^{2}}{2 t_{k-1}}+x_{B}-\sum_{i=k}^{n} t_{i}-\frac{t_{k-1}}{2}, \text { then } \\
R_{k}\left(t_{1}, \ldots, t_{n}\right) & =\sqrt{\left(\frac{a_{k}^{2}}{2 t_{k-1}}+\frac{t_{k-1}}{2}\right)^{2}+\left(y_{0}^{k}-y_{k}\right)^{2}}=R_{k}\left(t_{k-1}\right), \text { and } \\
\sin \beta_{k}\left(t_{1}, \ldots, t_{n}\right) & =\frac{y_{0}^{k}-y_{k-1}}{R_{k}\left(t_{k-1}\right)}=\sin \beta_{k}\left(t_{k-1}\right), \\
\cos \beta_{k}\left(t_{1}, \ldots, t_{n}\right) & =\frac{x_{0}^{k}\left(t_{1}, \ldots, t_{n}\right)-\left(x_{B}-\sum_{i=k-1}^{n} t_{i}\right)}{R_{k}\left(t_{k-1}\right)}=-\frac{a_{k}^{2}-t_{k-1}^{2}}{2 t_{k-1} R_{k}\left(t_{k-1}\right)}=\cos \beta_{k}\left(t_{k-1}\right), \\
\sin \mu_{k}\left(t_{1}, \ldots, t_{n}\right) & =\frac{y_{0}^{k}-y_{k}}{R_{k}\left(t_{k-1}\right)}=\sin \mu_{k}\left(t_{k-1}\right), \\
\cos \mu_{k}\left(t_{1}, \ldots, t_{n}\right) & =\frac{x_{B}-\sum_{i=k}^{n} t_{i}-x_{0}^{k}\left(t_{1}, \ldots, t_{n}\right)}{R_{k}\left(t_{k-1}\right)}=\frac{a_{k}^{2}+t_{k-1}^{2}}{2 t_{k-1} R_{k}\left(t_{k-1}\right)}=\cos \mu_{k}\left(t_{k-1}\right) .
\end{aligned}
$$

Thus, we see that $R_{k}, \beta_{k}$ and $\mu_{k}$ are functions of $t_{k-1}$ only, so for any other $i, i \neq(k-1)$, the partial derivatives of $\beta_{k}$ and $\mu_{k}$ with respect to $t_{i}$ are equal to zero. After differentiation and some 
simplifications we conclude that

$$
\frac{\beta_{k}^{\prime}\left(t_{k-1}\right)}{\sin \beta_{k}\left(t_{k-1}\right)}+\frac{\mu_{k}^{\prime}\left(t_{k-1}\right)}{\sin \mu_{k}\left(t_{k-1}\right)}=-\frac{1}{R_{k}\left(t_{k-1}\right)}, k=2, \ldots, n+1
$$

We find $\beta_{1}\left(t_{1}, \ldots, t_{n}\right)$ and $\mu_{1}\left(t_{1}, \ldots, t_{n}\right)$ in the similar way. The start point of the time-optimal trajectory is $A=\left(x_{A}, y_{0}\right)$ and the end point is $\left(x_{B}-\sum_{k=1}^{n} t_{i}, y_{1}\right)$. The only difference is that these functions depend on all $n$ variables $t_{1}, \ldots, t_{n}$. We find by differentiation that:

$$
\frac{\partial \mu_{1}}{\partial t_{i}} \frac{1}{\sin \mu_{1}\left(t_{1}, \ldots, t_{n}\right)}+\frac{\partial \beta_{1}}{\partial t_{i}} \frac{1}{\sin \beta_{1}\left(t_{1}, \ldots, t_{n}\right)}=\frac{1}{R_{1}\left(t_{1}, \ldots, t_{n}\right)}, i=1, \ldots, n \text {. }
$$

Hence, the stationary point of the function $t\left(t_{1}, \ldots, t_{n}\right)$ satisfies the following set of conditions:

$$
\begin{gathered}
\frac{\partial t\left(t_{1}, \ldots, t_{n}\right)}{\partial t_{k}}=-\left(\frac{1}{q_{1} R_{1}\left(t_{1}, \ldots, t_{n}\right)}\right)-\left(-\frac{1}{q_{k+1} R_{k+1}\left(t_{k}\right)}\right)=0, k=1, \ldots, n, \text { or equivalently } \\
q_{1} R_{1}\left(t_{1}, \ldots, t_{n}\right)=q_{2} R_{2}\left(t_{1}\right)=\ldots=q_{n+1} R_{n+1}\left(t_{n}\right)
\end{gathered}
$$

where $t_{i} \in\left(0, a_{i+1}\right]$ and $x_{B}-x_{A}-\sum_{i=1}^{n} t_{i}=t_{0}>0$.

It is possible to simplify the set of Equations (8) further: consider every consecutive pair of expressions $k$ and $k+1, k=1, \ldots, n$, and use the fact that the point $\left(x_{B}-\sum_{i=k}^{n} t_{i}, y_{k}\right)$ belongs at the same time to the circles with radii $R_{k}\left(t_{k-1}\right)$ and $R_{k+1}\left(t_{k}\right)$ as the time-optimal trajectory has to be continuous. We use the redundant variable $t_{0}$ to write down the system in the uniform way:

$$
\begin{aligned}
& q_{k+1}\left(a_{k+1}^{2}-t_{k}^{2}\right) t_{k-1}=q_{k}\left(a_{k}^{2}+t_{k-1}^{2}\right) t_{k}, k=1, \ldots, n, \\
& \sum_{i=0}^{n} t_{i}=x_{B}-x_{A}, \\
& t_{i} \in\left(0, a_{i+1}\right], i>0, \quad t_{0}>0 .
\end{aligned}
$$

Now, let us show that not more than one solution of the system of Equations (8) on the domain $\Pi_{i=1}^{n}\left(0, a_{i+1}\right]$ exists, hence system (9)-(11) also has not more than one solution. Suppose there exists a solution $\left(t_{1}^{*}, \ldots, t_{n}^{*}\right)$ of the system (8) with the corresponding trajectory $T$, see Figure 7 . Assume that there is another solution $\left(t_{1}^{\prime}, \ldots, t_{n}^{\prime}\right)$ with the corresponding trajectory $T^{\prime}$. The circle segments of both trajectories satisfy the system of Equations (8), so $q_{1} R_{1}\left(t_{1}^{*}, \ldots, t_{n}^{*}\right)=q_{2} R_{2}\left(t_{1}^{*}\right)=$ $\ldots=q_{n+1} R_{n+1}\left(t_{n}^{*}\right)$ and $q_{1} R_{1}\left(t_{1}^{\prime}, \ldots, t_{n}^{\prime}\right)=q_{2} R_{2}\left(t_{1}^{\prime}\right)=\ldots=q_{n+1} R_{n+1}\left(t_{n}^{\prime}\right)$. We denote $R_{i}\left(t_{1}^{*}, \ldots, t_{n}^{*}\right)$ as $R_{i}^{*}$ and $R\left(t_{1}^{\prime}, \ldots, t_{n}^{\prime}\right)$ as $R_{i}^{\prime}$.

Assume that $T^{\prime}$ is completely above $T$ and they intersect only in the end points $A$ and $B . K^{*}$ and $L^{*}$ are the points where $T$ intersects $y=y_{1}$ and $y=y_{n}$ respectively, $K^{\prime}$ and $L^{\prime}$ are the points where $T^{\prime}$ intersects $y=y_{1}$ and $y=y_{n}$ respectively. $K^{\prime}$ is to the left of $K^{*}$ and the left end $A$ of the trajectories $T$ and $T^{\prime}$ is fixed, so $R_{1}^{*}<R_{1}^{\prime} . L^{\prime}$ is to the left of $L^{*}$ and the right end $B$ of $T$ and $T^{\prime}$ is fixed, so $R_{n+1}^{*}>R_{n+1}^{\prime}$. Both $T$ and $T^{\prime}$ represent the solution of the system (8) so

$$
q_{n+1} R_{n+1}^{\prime}<q_{n+1} R_{n+1}^{*}=\ldots=q_{1} R_{1}^{*}<q_{1} R_{1}^{\prime}=\ldots=q_{n+1} R_{n+1}^{\prime},
$$

a contradiction. All other cases, like $T^{\prime}$ is completely below $T$ or they intersect in some intermediate point $M \neq B$, are proven in the same way. Therefore, not more than one solution of the system (8) exists. 


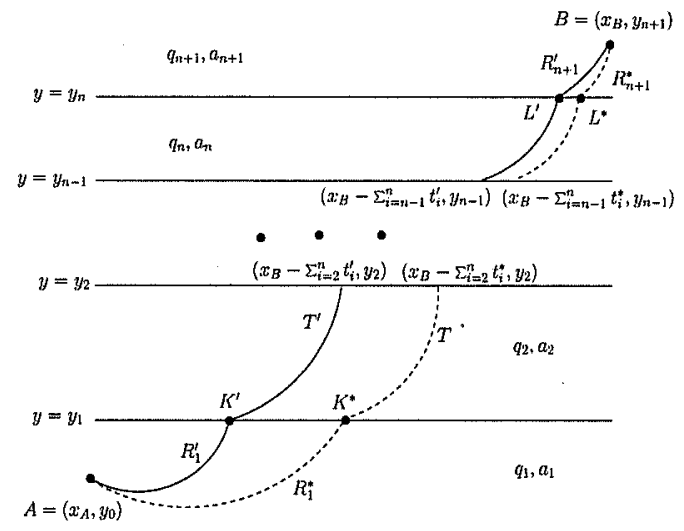

Fig. 7. Uniqueness of the solution

Furthermore, it is easy to check that if $t_{k} \in\left(0, a_{k+1}\right]$ is known then there exists not more than one value of $t_{k-1} \in\left(0, a_{k}\right]$, because two possible values of $t_{k-1}$ from Equation (9) are:

$$
t_{k-1}^{1,2}=\frac{q_{k+1}\left(a_{k+1}^{2}-t_{k}^{2}\right) \pm \sqrt{q_{k+1}^{2}\left(a_{k+1}^{2}-t_{k}^{2}\right)^{2}-4 q_{k}^{2} a_{k}^{2} t_{k}^{2}}}{2 q_{k} t_{k}}
$$

if the discriminant $q_{k+1}^{2}\left(a_{k+1}^{2}-t_{k}^{2}\right)^{2}-4 q_{k}^{2} a_{k}^{2} t_{k}^{2}$ is nonnegative. $t_{k-1} \leq a_{k}$, so only the solution with minus reminds, as otherwise the condition is broken. Hence, if the discriminant is nonnegative, there exists unique solution $t_{k-1}=t_{k-1}\left(t_{k}\right)$. So, given $t_{n}$ we can find $t_{k}, k=n-1, \ldots, 1$, using the next equation from the set, and either there exists the unique solution of the system or there is no solution, so the time-optimal trajectory which crosses $\left(x_{B}-t_{n}, y_{n}\right)$ and which is the set of circle segments does not exist.

Now, we have to solve the system of polynomial equations (9)-(11). We use the tools of computational algebraic geometry and commutative algebra to solve the system, see, e.g., [11]. The brief introduction into the theory is given in Appendix C. In particular, the Elimination Theorem guaranties that we can find a polynomial consequence of the system (9)-(11) in finitely many steps, such that it contains only $t_{n}$ as a variable. There are numerical methods to find the roots of such an equation with high precision (for example, the Jenkins-Traub algorithm, see [21]). If there exists a root $t_{n}^{*} \in\left(0, a_{n+1}\right]$ then we can find all other values of $t_{k}^{*}$ from Equation (12). Otherwise there is no time-optimal trajectory which is a set of circle segments.

There are some practical problems with the method suggested above, see [17,22]. Computing the roots of the univariate polynomial can be ill-conditioned for polynomials of degree greater than 14,15 , while Groebner basis can contain as a consequence a polynomial at one variable of high degree. In fact, for our case the polynomial consequence, which contains only $t_{n}$, is a crucial one. All other values of $t_{i}, i<n$, can be found directly from the quadratic Equation (12). As an example, if the velocity function has only one breakpoint then the highest degree of the polynomial at $t_{n}$ is 3 (hence, it is solvable analytically), for two breakpoints the highest degree is 7 . So, if the number of breakpoints is small then the method is applicable.

\subsection{Circle and line segments}

In this subsection we briefly sketch the search of the potential time-optimal solution which contains circle and straight line segments. In fact, we reduce this case to the previous one, which contains 
only circle segments. The straight line segment appears when $t_{k}$ is equal to zero, so we have $\sum_{i=0}^{n}\left(\begin{array}{l}n \\ i\end{array}\right)=2^{n}$ combinations of possible set of zero $t_{k}$ 's. We assume that the number of breakpoints of the velocity function $n$ is constant, so $2^{n}$ is a constant factor.

Assume that only one $t_{k}$ is equal to zero, then the time needed to go alone the trajectory which contains circle segments and one straight line segment is:

$$
t\left(t_{1}, \ldots, t_{n}\right)=\sum_{i=1, \ldots, k, k+2, \ldots, n} \int_{0}^{\gamma_{i}\left(t_{1}, \ldots, t_{n}\right)} \frac{d \alpha}{\sin \left(\alpha+\beta_{i}\left(t_{1}, \ldots, t_{n}\right)\right)}+t_{\text {line }}
$$

where $t_{\text {line }}$ is the time to go alone the vertical line segment within the layer $L_{k}$ from the altitude $y=y_{k}$ to the altitude $y=y_{k+1}$. It is a constant with respect to the variables $t_{1}, \ldots, t_{k-1}, t_{k+1}, \ldots, t_{n}$.

We find the stationary point conditions for Equation (13) in the similar way as in Subsection 4.1. The partial derivative of the term $t_{\text {line }}$ with respect to any $t_{i}, i \neq k$, is equal to zero, as it is a constant. The functions $\beta_{i}\left(t_{1}, \ldots, t_{k-1}, t_{k+1}, \ldots, t_{n}\right)$ and $\mu_{i}\left(t_{1}, \ldots, t_{k-1}, t_{k+1}, \ldots, t_{n}\right), i \neq(k+1)$, are still the functions at $t_{k-1}$ only, as we do the same steps, replacing $t_{k}$ with zero. $\beta_{k+1}$ and $\mu_{k+1}$ are not determined any more. So we end up with the similar system of equations:

$$
q_{1} R_{1}\left(t_{1}, \ldots, t_{k-1}, t_{k+1}, \ldots, t_{n}\right)=q_{i} R_{i}\left(t_{i-1}\right), i=1, \ldots, k, k+2, \ldots, n .
$$

If more than one of the $t_{k}$ 's are equal to zero, then Equation (13) contains more constant factors, but the rest of the analysis remains the same. Finally, we choose the best trajectory with respect to time (determined by Equation (13)) among the trajectories generated by some set of $t_{i}$ 's which are equal to zero. The case when the trajectory contains only circle segments is also included, as it is generated by the empty set of zero $t_{i}$ 's.

\section{Conclusion}

In this paper we have addressed the problem of determining a time-optimal helicopter trajectory between two points in two-dimensional space, where the speed of the helicopter depends on the altitude. We have characterized the time-optimal trajectories for the basic problem, where the speed decreases linearly in altitude. We used the motions primitives to solve the basic helicopter problem with rectilinear obstacles in $2 \mathrm{D}$ in polynomial time and to reduce the general helicopter problem with piece-wise linear velocity function to the set of multivariate polynomial equations. If the number of velocity function breakpoints is constant then it enables us to solve the general helicopter problem in polynomial time using the algebraic elimination theory.

There are several interesting research directions that can be followed. First, it is an open question whether the general helicopter problem with polygonal obstacles in $2 \mathrm{D}$ admits a polynomial time algorithm. Second, are there efficient polynomial time approximation schemes for the general helicopter problem with obstacles in 3D? Notice, the three-dimensional problem with obstacles is NP-hard as it generalizes the Euclidean shortest-path problem with obstacles in 3D.

\section{Acknowledgments}

We would like to thank Erik Balder for his very useful comments and links. 


\section{References}

1. P.K. Agarwal, S. Har-Peled, M. Sharir, and K.R. Varadarajan. Approximating Shortest Paths on a Convex Polytope in Three Dimensions. JACM, 44(4), 567-584, 1997.

2. P.K. Agarwal, P. Raghavan, and H. Tamaki. Motion planning for a steering constrained robot through moderate obstacles. In Proc. ACM Symposium on Computational Geometry, 343-352, 1995.

3. Basic helicopter handbook. US Department of Transportation, Federal Aviation Administration, Advisory Circular 61-13A, 1973.

4. D.P. Bertsekas. Dynamic Programming and Optimal Control. Athena Scientific, 1995.

5. J.T. Betts. Practical Methods for Optimal Control Using Nonlinear Programming. SIAM Press, Philadelphia, Pennsylvania, 2001

6. J.T. Betts. Survey of Numerical Methods for Trajectory Optimization. J. of Guidance, Control, and Dynamics, 21(2), 193-207, 1998.

7. J. Canny and J.H. Reif. New lower bound techniques for robot motion planning problems. In Proc. of the 28th Annual IEEE Symposium on Foundations of Computer Science, IEEE, 49-60, 1987.

8. H. Chitsaz and S.M. LaValle. Minimum wheel-rotation paths for differential drive mobile robots among piecewise smooth obstacles. In Proc. IEEE International Conference on Robotics and Automation, IEEE, 2718-2723, 2007.

9. H. Chitsaz, S.M. LaValle, and D.J. Balkcom. Minimum Wheel-Rotation Paths for Differential-Drive Mobile Robots. The International Journal of Robotics Research, 28(1), 66-80, 2009.

10. H. Chitsaz, S.M. LaValle, D.J. Balkcom, and M.T. Mason. An explicit characterization of minimum wheel-rotation paths for differential-drives. In Proc. IEEE International Conference on Methods and Models in Automation and Robotics, IEEE, 1616-1623, 2006.

11. D. Cox, J. Little, and D. O'Shea. Ideals, Varieties and Algorithms. Springer Verlag, New York, 1992.

12. O. Daescu, J. S. B. Mitchell, S. Ntafos, J. D. Palmer and C. K. Yap. k-link shortest paths in weighted subdivisions. In Proc. International Workshop on Algorithms and Data Structures, 325-327, 2005.

13. O. Daescu, J. S. B. Mitchell, S. Ntafos, J. D. Palmer and C. K. Yap. Approximating minimum-cost polygonal paths of bounded number of links in weighted subdivisions. In Proc. ACM Symposium on Computational Geometry, 483-484, 2006.

14. I.M. Gelfand and S.V. Fomin. Calculus of Variations. Dover Publications, Inc., NY, 2000.

15. I.M. Gelfand and S.V. Fomin. Calculus of Variations. Gos. Izd. fiz.-mat. lit., Moscow, 1961.

16. M. Kline. Calculus: an intuitive and physical approach. Dover Publications, Inc., New York, 1998.

17. D. Manocha. Solving systems of polynomial equations. IEEE Comput. Graph. Appl. 14:46-55, 1994.

18. J.S.B. Mitchell and C.H. Papadimitriou. Planning shortest paths. In Proc. SIAM Conference on Geometric Modeling and Robotics, 1-21, 1985.

19. C.H. Papadimitriou and K. Steiglitz. Combinatorial optimization: algorithms and complexity. Prentice-Hall, Inc., Upper Saddle River, NJ, USA, 1982.

20. L.S. Pontryagin, V.G. Boltyanskii, R.V. Gamkrelidze, and E.F. Mishchenko. The Mathematical Theory of Optimal Processes. John Wiley \& Sons Inc, 1962.

21. W.H. Press, S.A. Teukolsky, W.T. Vetterling and B.P. Flannery. Numerical recipes in C. The art of scientific computing. 2nd ed. Cambridge University Press, Cambridge. 1992.

22. J.H. Wilkinson. The evaluation of the zeros of ill-conditioned polynomials. Parts $i$ and ii. Numer. Math. 1:150$180,1959$. 


\section{A Solution to the basic helicopter problem}

In this section we derive motion primitives to the basic helicopter problem.

Theorem 2. Let $C$ be the intersection point of the line $y=\frac{v_{0}}{q}$ and the line equidistant from $A$ and $B$. The segment $T_{A B}$ of the circle with center $C$ and radius $R=|C A|=|C B|$ is a time-optimal trajectory between $A$ and $B$. The time needed to travel along $T_{A B}$ is

$$
t_{o p t}=t_{T_{A B}}=\frac{1}{q} \int_{0}^{\gamma} \frac{d \alpha}{\sin (\alpha+\beta)}=\frac{1}{q} \ln \left|\frac{\tan \frac{\beta}{2}+\tan \frac{\gamma}{2}}{\tan \frac{\beta}{2}-\tan ^{2} \frac{\beta}{2} \tan \frac{\gamma}{2}}\right|,
$$

where $\beta$ is the angle between $C A$ and the $x$-axis, and $\gamma$ is the angle between $C A$ and $C B$.

Proof. The beginning of the proof is given in Section 2. The following integral is derived to calculate the time needed to go alone the arbitrary convex trajectory $T$ :

$$
t_{T}=\int_{0}^{\gamma} \frac{\sqrt{R^{\prime 2}(\alpha)+R^{2}(\alpha)} d \alpha}{q R(\alpha) \sin (\alpha+\beta)}=\frac{1}{q} \int_{0}^{\gamma} \sqrt{\left(\frac{R^{\prime}(\alpha)}{R(\alpha)}\right)^{2}+1} \frac{d \alpha}{\sin (\alpha+\beta)} .
$$

Notice, this integral is well defined as $0<\alpha+\beta<\pi$ for any $\alpha \in[0, \gamma](\beta$ and $\gamma$ are two of the three angles of the triangle). Furthermore, for the trajectory $T$ we have that $R(0)=R(\gamma)=R=$ $|C A|=|C B|$, as the point $C$ is equidistant from $A$ and $B$.

Now, we replace $R(\alpha)$ with the function $f(\alpha)=\ln (R(\alpha))$. Since $R(\alpha)$ is continuously differentiable, the function $f(\alpha)$ is continuously differentiable as well. Furthermore, $f^{\prime}(\alpha)=\frac{R^{\prime}(\alpha)}{R(\alpha)}$, and the following boundary conditions hold: $f(0)=f(\gamma)=\ln R$, where $R=|C A|=|C B|$. For the function $f(\alpha)$ the travel time is calculated as follows:

$$
t_{T}=\frac{1}{q} \int_{0}^{\gamma} \sqrt{1+f^{\prime 2}(\alpha)} \frac{d \alpha}{\sin (\alpha+\beta)}
$$

Now, we have to find all minimizers of the functional (15). The following theorem of the calculus of variation is used:

Theorem 3 ([15], p. 21; see also [14]). Let $S(y)=\int_{a}^{b} F\left(t, y(t), y^{\prime}(t)\right) d t$ be a functional, where $y:[a, b] \subset \mathbb{R} \rightarrow X$ is differentiable and $y(a)=y_{a}, y(b)=y_{b}, y^{\prime}$ is the continuous derivative of $y$ and $F$ is a real-valued function with continuous first partial derivatives. If the function $y(t)$ is a stationary point of the functional $S(y)$, then it satisfies the equation:

$$
F_{y}^{\prime}\left(t, y(t), y^{\prime}(t)\right)-\frac{d}{d t} F_{y^{\prime}}^{\prime}\left(t, y(t), y^{\prime}(t)\right)=0
$$

Notice that the trajectory is a circle segment if and only if the radius-vector $R(\alpha)$ is constant, which is possible if and only if the function $f(\alpha)=\ln (R(\alpha))$ is also a constant. Therefore, we can concentrate on Equation (15) proving that $f(\alpha)=$ const is the unique minimizer of the functional (15).

1) We prove first that the circle segment with the center $C$ and radius $R=|C A|$ is a time-optimal trajectory.

Consider an arbitrary trajectory $T . f^{\prime 2}(\alpha) \geq 0$ for any $T$, therefore, the following lower bound exists:

$$
t_{T} \geq \frac{1}{q} \int_{0}^{\gamma} \frac{d \alpha}{\sin (\alpha+\beta)}
$$


On the other hand, the last integral represents the time needed to travel along the circle segment $T_{A B}$ with the center $C$ and the constant radius $R=|C A|$. Therefore, the derived circle segment is at least as good as any other trajectory.

2) Now, we show that $T_{A B}$ is the unique time-optimal trajectory in the class of continuously differentiable functions. Consider the functional (15) with the integrand $F\left(\alpha, f, f^{\prime}\right)=\frac{\sqrt{1+f^{\prime 2}(\alpha)}}{\sin (\alpha+\beta)}$. Suppose $f(\alpha)$ is the minimizer of the functional. Therefore, the Euler-Lagrange Equation (16) holds for $f(\alpha)$, because it is a necessary condition for the stationary point of any functional. We obtain from Equation (16) that $\frac{d}{d \alpha}\left(F_{f^{\prime}}^{\prime}\right)=0$ as $F_{f}^{\prime}=0$. Hence,

$$
F_{f^{\prime}}^{\prime}=\frac{f^{\prime}(\alpha)}{\sqrt{1+f^{\prime 2}(\alpha)} \sin (\alpha+\beta)}=\text { const. }
$$

Since $\sqrt{1+f^{\prime 2}(\alpha)} \geq 1$ and $\sin (\alpha+\beta)>0,(0<\alpha+\beta<\pi)$, the denominator in Equation (17) is always positive. So the sign of $f^{\prime}(\alpha)$ depends only from the sign of the constant. If const $=0$ then $f^{\prime}(\alpha)=0$ and $f(\alpha)$ is a constant function, so as $R(\alpha)$. This minimizer is the circle segment described above. If const $>0(<0)$ then $f(\alpha)$ is strictly increasing (decreasing), but this is the contradiction to the boundary condition $f(0)=f(\gamma)=\ln R$.

Hence, there are no other time-optimal trajectories but the circle segment $T_{A B}$ with the center $C$ and the radius $R=|C A|=|C B|$.

The time needed to travel along the circle segment $T_{A B}$ can be computed as the value of the integral:

$$
\begin{aligned}
t_{\text {opt }} & =\frac{1}{q} \int_{0}^{\gamma} \frac{d \alpha}{\sin (\alpha+\beta)}=\frac{1}{q} \int_{\beta}^{\beta+\gamma} \frac{d \tau}{\sin \tau}=\frac{1}{q} \int_{\tan \frac{\beta}{2}}^{\tan \frac{\beta+\gamma}{2}} \frac{d t}{t}= \\
& =\frac{1}{q} \ln \left|\frac{\tan \frac{\beta+\gamma}{2}}{\tan \frac{\beta}{2}}\right|=\frac{1}{q} \ln \left|\frac{\tan \frac{\beta}{2}+\tan \frac{\gamma}{2}}{\tan \frac{\beta}{2}-\tan ^{2} \frac{\beta}{2} \tan \frac{\gamma}{2}}\right| .
\end{aligned}
$$

\section{B Potential breakpoints, one step obstacle case}

Lemma 4. For three consecutive points $A=\left(x_{1}, y_{1}\right), E=\left(x_{2}, y_{1}\right)$ and $B=\left(x_{2}, y_{2}\right)$ from $S$, such that $y_{1}<y_{2}$, the time-optimal trajectory from $A$ to $B$ with obstacles is:

a) if $C_{A B}$ is to the left of $A$ then $T_{A B}$ is a time-optimal trajectory,

b) if $C_{A B}$ is to the right of $A$ then time-optimal trajectory is the combination of the straight horizontal line segment $L_{A D}$ and the circle segment $T_{D B}$, where $D$ and $C_{D B}$ determine a vertical line. In particular,

$$
D=\left(x_{2}-\sqrt{\left(y_{2}-y_{1}\right)\left(2 y_{0}-y_{1}-y_{2}\right)}, y_{1}\right) \text {. }
$$

Proof. a) Follows directly from Theorem 1 , as $T_{A B}$ does not hit the horizontal and vertical obstacles $L_{A E}$ and $L_{E B}$.

b) $T_{A B}$ hits $L_{A E}$ as $C_{A B}$ is to the right of $A$. Let us find the first point $D=\left(x, y_{1}\right)$ to the right of $A$ such that $T_{D B}$ does not hit the obstacle $L_{A E}$. The circle does not hit $L_{A E}$ for the first time when $L_{A E}$ is the tangent line of $T_{D B}$. So the $x$-coordinate of the circle center $C_{D B}$ and $D$ are the same. Hence, $R_{D B}=d\left(C_{D B}, D\right)=d\left(C_{D B}, B\right)$ and $C_{D B}$ has coordinates $\left(x, y_{0}\right)$. We have the following equation: 


$$
\begin{aligned}
(x-x)^{2} & +\left(y_{0}-y_{1}\right)^{2}=\left(x_{2}-x\right)^{2}+\left(y_{0}-y_{2}\right)^{2}, \\
\left(x-x_{2}\right)^{2} & =\left(y_{2}-y_{1}\right)\left(2 y_{0}-y_{1}-y_{2}\right) \geq 0, \text { as } y_{0} \geq y_{2}>y_{1}, \\
x & =x_{2}-\sqrt{\left(y_{2}-y_{1}\right)\left(2 y_{0}-y_{1}-y_{2}\right)}, \text { as } x \leq x_{2} .
\end{aligned}
$$

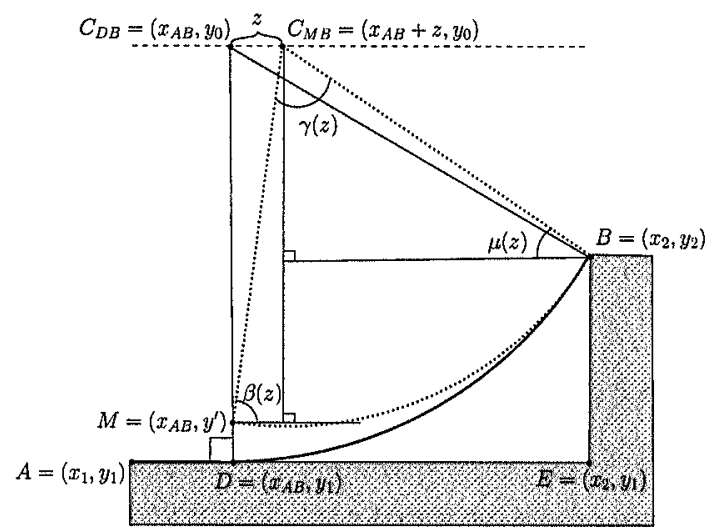

Fig. 8. The optimal trajectory for the one step case, $T_{A B}$ hits $L_{A E}$

Here and below we denote the found value of $x$ for $A$ and $B$ as $x_{A B}$. We show now that the constructed trajectory $L_{A D}$ plus $T_{D B}$ is time-optimal with obstacles. Suppose there exists another trajectory $T$ such that $t_{T}<t_{L_{A D}}+t_{T_{D B}}$. $T$ has to cross the line $x=x_{A B}$ at some altitude above $y_{1}$ to avoid the obstacle $L_{A E}$, for example at point $M=\left(x_{A B}, y^{\prime}\right), y^{\prime} \in\left[y_{1}, y_{0}\right]$, see Figure 8. $M$ splits the trajectory $T$ into two parts:

- $A M$ such that $t_{A M} \geq t_{L_{A D}}$, the proof is the same as the proof of Lemma 1 ,

- $M B$, the trajectory $M B$ is unknown, but the best we can do is to go from $M$ to $B$ along $T_{M B}$. Let us show that $t_{T_{M B}} \geq t_{T_{D B}}$.

The position of the point $C_{M B}$ is a one-to-one function of the position of the point $M$. For the simplicity we consider $z$ as a parameter, where $C_{M B}=\left(x_{A B}+z, y_{0}\right)$. By Theorem 1 the time needed to go along $T_{M B}$ is given by the following integral:

$$
t_{T_{M B}}(z)=\frac{1}{q} \int_{0}^{\gamma(z)} \frac{d \alpha}{\sin (\alpha+\beta(z))} .
$$

We show that the derivative of $t_{T_{M B}}(z)$ is positive and thus the function $t_{T_{M B}}(z)$ is monotonically increasing:

$$
\begin{aligned}
\frac{d}{d z}\left(q t_{T_{M B}}(z)\right) & =\int_{0}^{\gamma(z)}-\frac{\cos (\alpha+\beta(z)) \beta^{\prime}(z) d \alpha}{\sin ^{2}(\alpha+\beta(z))}+\frac{\gamma^{\prime}(z)}{\sin (\gamma(z)+\beta(z))}= \\
& =-\beta^{\prime}(z) \int_{\sin \beta(z)}^{\sin (\beta(z)+\gamma(z))} \frac{d \tau}{\tau^{2}}+\frac{\gamma^{\prime}(z)}{\sin (\gamma(z)+\beta(z))}= \\
& =\frac{\beta^{\prime}(z)+\gamma^{\prime}(z)}{\sin (\gamma(z)+\beta(z))}-\frac{\beta^{\prime}(z)}{\sin \beta(z)}=-\left[\frac{\beta^{\prime}(z)}{\sin \beta(z)}+\frac{\mu^{\prime}(z)}{\sin \mu(z)}\right]
\end{aligned}
$$


where $\mu(z)=\pi-\gamma(z)-\beta(z)$ is the angle between $C_{M B} B$ and the horizontal axis. $R_{M B}=$ $R(z)=\sqrt{\left(x_{A B}+z-x_{2}\right)^{2}+\left(y_{0}-y_{2}\right)^{2}}$, then $\cos \beta(z)=\frac{z}{R(z)}, \sin \beta(z)=\frac{\sqrt{R^{2}(z)-z^{2}}}{R(z)}, \cos \mu(z)=$ $\frac{x_{2}-x_{A B}-z}{R(z)}, \sin \mu(z)=\frac{y_{0}-y_{2}}{R(z)}$. Hence we obtain,

$$
\frac{d}{d z}\left(q t_{T_{M B}}(z)\right)=-\left[\frac{\beta^{\prime}(z)}{\sin \beta(z)}+\frac{\mu^{\prime}(z)}{\sin \mu(z)}\right]=\frac{z\left(x_{2}-x_{A B}\right)}{R(z)\left(R^{2}(z)-z^{2}\right)} \geq 0,
$$

as $x_{2}>x_{A B}$ and the denominator is positive, so the time increases with the growth of $z$. $\frac{d}{d z}\left(q t_{T_{M B}}(z)\right)=0$ if and only if $z=0$, which means $C_{M B}=C_{D B}$. We can conclude that $t_{T_{D B}}$ is smaller than $t_{M B}$ for any trajectory $M B$.

So, the constructed trajectory $L_{A D}$ plus $T_{D B}$ is the time-optimal for one step as

$$
t_{L_{A D}}+t_{T_{D B}} \leq t_{A M}+t_{T_{M B}} \leq t_{T}
$$

\section{Dynamic programming to the basic helicopter problem with rectilinear obstacles}

In this appendix we prove the set of observations used in Lemma 3 and the lemma itself.

Observation 4 For any three points $A=\left(x_{1}, y_{1}\right), B=\left(x_{2}, y_{2}\right)$ and $M=\left(x_{3}, y_{3}\right)$ such that $x_{1}<$ $x_{3}<x_{2}$ and $y_{3}<y_{1}, y_{3}<y_{2}, T_{A B}$ lies above $T_{A M}$ and $T_{M B}$ on the interval $\left[x_{1}, x_{2}\right]$.

Proof. Denote the $x$-coordinates of the centers of the circle segments $T_{A M}, T_{M B}$ and $T_{A B}$ as $x_{0}^{A M}, x_{0}^{M B}$ and $x_{0}^{A B}$ respectively. Then $x_{0}^{M B} \leq x_{0}^{A B} \leq x_{0}^{A M}$ as $x_{0}^{A B}=\lambda x_{0}^{M B}+(1-\lambda) x_{0}^{A M}$ for $\lambda=\frac{x_{2}-x_{3}}{x_{2}-x_{1}} \in[0,1]$.

The circle segment $T_{A B}$ is given by the equation $\left(x-x_{0}^{A B}\right)^{2}+\left(y-y_{0}\right)^{2}=R_{A B}^{2}$, where $R_{A B}^{2}=$ $\left(x_{0}^{A B}-x_{1}\right)^{2}+\left(y_{0}-y_{1}\right)^{2}$, so $y=y_{0}-\sqrt{R_{A B}^{2}-\left(x-x_{0}^{A B}\right)^{2}}$. Similarly, the circle segment $T_{A M}$ is given by the equation $y=y_{0}-\sqrt{R_{A M}^{2}-\left(x-x_{0}^{A M}\right)^{2}}$, where $R_{A M}^{2}=\left(x_{0}^{A M}-x_{1}\right)^{2}+\left(y_{0}-y_{1}\right)^{2}$. The circle segment $T_{A B}$ is above the circle segment $T_{A M}$ if $y_{0}-\sqrt{R_{A B}^{2}-\left(x-x_{0}^{A B}\right)^{2}}$ is bigger than $y_{0}-\sqrt{R_{A M}^{2}-\left(x-x_{0}^{A M}\right)^{2}}$ for any $x \in\left[x_{1}, x_{2}\right]$. It is enough to compare the expressions under the radical to verify this fact:

$$
\begin{aligned}
& R_{A B}^{2}-\left(x-x_{0}^{A B}\right)^{2}-\left(R_{A M}^{2}-\left(x-x_{0}^{A M}\right)^{2}\right)= \\
& \left(x_{0}^{A B}-x_{1}\right)^{2}+\left(y_{0}-y_{1}\right)^{2}-\left(x-x_{0}^{A B}\right)^{2}-\left(x_{0}^{A M}-x_{1}\right)^{2}-\left(y_{0}-y_{1}\right)^{2}+\left(x-x_{0}^{A M}\right)^{2}= \\
& 2\left(x_{0}^{A B}-x_{0}^{A M}\right)\left(x-x_{1}\right) \leq 0 .
\end{aligned}
$$

So $\sqrt{R_{A B}^{2}-\left(x-x_{0}^{A B}\right)^{2}} \leq \sqrt{R_{A M}^{2}-\left(x-x_{0}^{A M}\right)^{2}}$ for any $x \in\left[x_{1}, x_{2}\right]$, hence, $y_{0}-\sqrt{R_{A B}^{2}-\left(x-x_{0}^{A B}\right)^{2}} \geq$ $y_{0}-\sqrt{R_{A M}^{2}-\left(x-x_{0}^{A M}\right)^{2}}$. Therefore, $T_{A B}$ is higher than $T_{A M}$. The proof that $T_{A B}$ is higher than $T_{M B}$ is similar.

Observation 5 For any three consecutive points $A=\left(x_{1}, y_{1}\right), C=\left(x_{2}, y_{1}\right)$ and $B=\left(x_{2}, y_{2}\right)$ such that $x_{1}<x_{2}$ and $T_{A B}$ hits $L_{A C}$, the time-optimal trajectory lies above $T_{A B}$ on the interval $\left[x_{1}, x_{2}\right]$. 
Proof. We consider $x_{1}=0$ and $D=\left(\Delta x, y_{1}\right)$ without loss of generality, where $\Delta x$ is determined by Lemma $2 \mathrm{~b}$ ) as in this case $L_{A D}$ plus $T_{D B}$ is the time-optimal trajectory with one step obstacle. Furthermore, $\Delta x=x_{0}^{D B}$. Then by Equation (5)

$$
\begin{aligned}
& x_{0}^{A B}=\frac{-2\left(y_{2}-y_{1}\right) y_{0}-y_{1}^{2}+x_{2}^{2}+y_{2}^{2}}{2 x_{2}}, \\
& x_{0}^{D B}=\Delta x=\frac{-2\left(y_{2}-y_{1}\right) y_{0}-y_{1}^{2}+x_{2}^{2}+y_{2}^{2}}{2\left(x_{2}-\Delta x\right)}+\frac{(\Delta x)^{2}}{2\left(\Delta x-x_{2}\right)},
\end{aligned}
$$

so $2 x_{0}^{D B}\left(x_{2}-\Delta x\right)+(\Delta x)^{2}=2 x_{2} x_{0}^{A B}$, hence $x_{0}^{D B}-x_{0}^{A B}=\Delta x-x_{0}^{A B}=\frac{(\Delta x)^{2}}{2 x_{2}} \geq 0$. Hence, $x_{0}^{D B} \geq x_{0}^{A B}$, so the circle segment $T_{D B}$ is above $T_{A B}$ for any $x \in\left[x_{1}, x_{2}\right]$, the proof of this fact is similar to the proof of Observation 1.
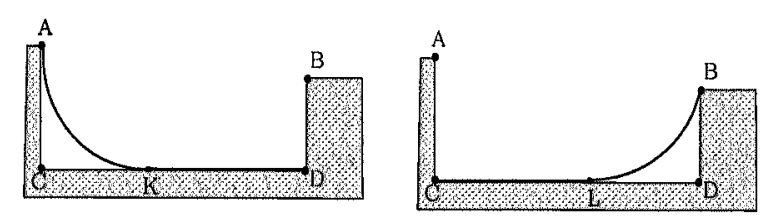

Fig. 9. The time-optimal trajectory for the unique horizontal obstacle between $A$ and $B$

Observation 6 Given four consecutive points $A=\left(x_{1}, y_{1}\right), C=\left(x_{1}, y_{2}\right), D=\left(x_{2}, y_{2}\right)$ and $B=$ $\left(x_{2}, y_{3}\right)$ from $S$ such that $x_{1}<x_{2}, y_{2}<y_{1}, y_{2}<y_{3}$ and $T_{A B}$ hits $L_{C D}$, the time-optimal trajectory with obstacles is the combination of the circle segment $T_{A K}$, straight line $L_{K L}$ and circle segment $T_{L B}$, where $K$ and $L$ are the points of intersection of $T_{A D}$ and $T_{C B}$ with $L_{C D}$ respectively.

Proof. We consider the triplets $A, C, D$ and $C, D, B$ as the input to Lemma 2 (see Figure 9). $T_{A K}$ does not intersect $T_{L B}$ by Observation 1 , as $T_{A B}$ hits $L_{C D}$.

Lemma 5. If the time-optimal trajectory $T_{A B}$ between any two potential breakpoints $A=\left(x_{1}, y_{1}\right), B=$ $\left(x_{2}, y_{2}\right), \quad x_{1}<x_{2}$ hits the obstacles, then there exists a potential breakpoint $C$ between $A$ and $B$ such that the time-optimal trajectory with obstacles from $A$ to $B$ passes $C$.

Proof. Assume the statement is not correct, hence the trajectory hits some obstacle and there exists a time-optimal trajectory $T$ with obstacles from $A$ to $B$ such that it does not hit any obstacle and does not pass any potential breakpoint.

We split the proof into five parts depending on the type of "terrain" between $A$ and $B$.

1) The obstacles between $A$ and $B$ are monotonically increasing (or decreasing), see Figure 10.

First we notice that $T$ is convex, otherwise we can always locally shortcut it with the straight line in such a way that it still does not pass any potential breakpoint, but the straight line is always better than any trajectory above it by Lemma 1 , so $T$ is not time-optimal.

We assume without loss of generality that $y_{1}<y_{2}$. Let us consider the last obstacle $C=\left(x_{3}, y_{3}\right)$ on the way along $T, T$ does not pass $C$ by assumption. By Lemma 2 the time-optimal trajectory from $C$ to $B$ has one of two shapes: 


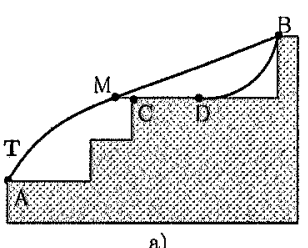

a)

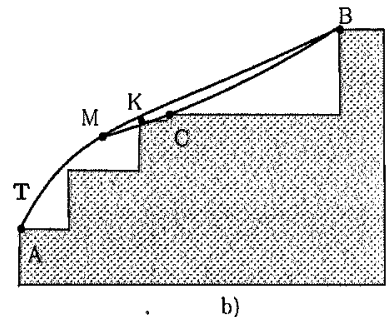

b)

Fig. 10. Monotonic obstacle case

- it is the combination of the straight line $L_{C D}$ and $T_{D B}$, see Figure 10a). The intersection of the line $y=y_{3}$ and the trajectory $T$ is point $M$. The time-optimal trajectory from $M$ to $B$ is the combination of the straight line $L_{M D}$ and the circle segment $T_{D B}$ by Lemma $2 \mathrm{~b}$ ). Then the following combined trajectory is better than $T: T$ before the point $M$, straight line $L_{M D}$ and $T_{D B}$, and it passes the potential breakpoint $C$, a contradiction.

- it is $T_{C B}$, see Figure 10b). $M$ is the point of intersection of $T$ and $T_{C B}$. If $T_{C B}=T_{M B}$ does not hit any obstacle before crossing $T$ or it just crosses the obstacle in the potential breakpoint $K$ then the trajectory $T$ between $A$ and $M$ plus $T_{M B}$ is better than $T$ and it passes the potential breakpoint $C$, a contradiction.

If $T_{M B}$ hits the obstacle which is determined by the potential breakpoint $K$ then we consider $T_{K B}$ (or $L_{K D_{K}}$ and $T_{D_{K} B}$ ) by Lemma $2 \mathrm{a}$ ) (or b)) and so on. We have only a finite number of obstacles so finally we can find the "last" obstacle $L$ such that the time-optimal trajectory from Lemma 2 does not hit this obstacle. Any new circle segment or combination of the line and circle segment lies higher than the previous one by induction, so it does not hit any obstacle to the right of the considered potential breakpoint. We use the time-optimal trajectory which is determined by the potential breakpoint $L$ and $B$ to shortcut $T$ as in the previous case, a contradiction.

2) There is an obstacle between $A$ and $B$ which is higher than $A$ and $B$, see Figure 11. By Lemma 1 we can always shortcut $T$ with the line $L_{K M}$ which passes 2 potential breakpoints, a contradiction.

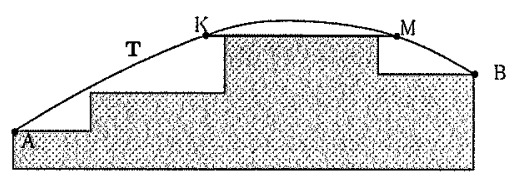

Fig. 11. Concave obstacle case

3) The obstacles between $A$ and $B$ is a convex rectilinear function, see Figure 12. Similar to case 1) the trajectory $T$ is convex. Denote the lowest point of the trajectory $T$ as point $M=\left(x_{M}, y_{M}\right)$. We consider two following cases:

- All the obstacles which are hit by $T_{A B}$ are lower than $M$, see Figure 12a). We introduce a new instance: the horizonal line $y=y^{\prime}$ at the altitude of the highest obstacle $K=\left(x^{\prime}, y^{\prime}\right)$, hit by $T_{A B} . T$ is a valid trajectory for the new instance. The time-optimal trajectory $T^{\prime}$ for the new instance by Observation 3 is the circle segment $T_{A K_{1}}$, line segment $L_{K_{1} K_{2}}$ and circle 
segment $T_{K_{2} B}$, where $K_{1}, K_{2}$ belongs to the line $y=y^{\prime}$. By Observation 2 this trajectory does not hit any obstacle of the original instance, so it is a valid trajectory for the original instance. $T^{\prime}$ is time-optimal for the new instance, hence it is better than $T$. So, $T^{\prime}$ is also better than $T$ for the original instance, a contradiction.

- There is at least one obstacle $K=\left(x^{\prime}, y^{\prime}\right)$, which is hit by $T_{A B}$, and it is higher than $M$, see Figure 12b). We introduce a new instance: the horizontal line $y=y_{M}$. If either $T_{A M}$ or $T_{M B}$ hits the obstacles then we are in the settings of the case 1) for one of the segments $T_{A M}$ or $T_{M B}$, hence there is a trajectory $T^{\prime}$ which is better than $T$ and it passes one of the potential breakpoints.

So we only consider the case when $T_{A M}$ and $T_{M B}$ do not hit any obstacle. It means that $T$ is just a combination of $T_{A M}$ and $T_{M B}$. By Observation $1 T_{A B}$ is above $T$, so $T_{A B}$ also does not hit any obstacle, a contradiction.

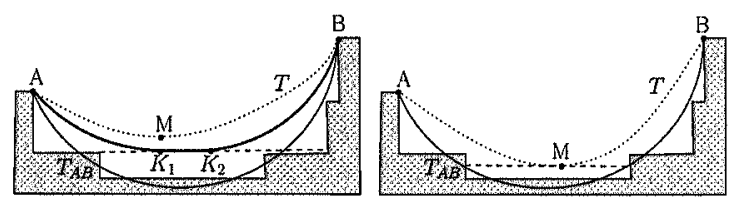

a)

b)

Fig. 12. Convex obstacle case

4) There is an obstacle between $A$ and $B$ which is higher than $A$, lower than $B$ and it is the point of non-monotonicity, see Figure 13. If the trajectory $T$ "goes down" after this obstacle, see Figure 13a), we can shortcut it as in case 2), a contradiction. If it stays higher, see Figure 13b), then we can introduce a new equivalent monotonic instance as in case 1).

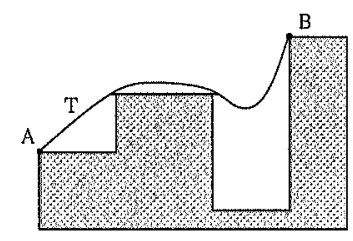

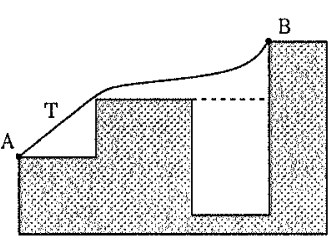

b)

Fig. 13. Non-monotonic obstacle case, the obstacle is higher than $A$

5) The obstacles between $A$ and $B$ are not presented by a convex rectilinear function, and all the obstacles are lower than $A$ and $B$, see Figure 14. The obstacle function is not convex, so there exists an obstacle which is concave. If $T$ "goes down" on both sides of this obstacle we can shortcut is as in case 2), see Figure 14a), a contradiction. If $T$ stays higher at least on one side, see Figure 14b), then we can introduce a new convex instance as in case 4 ), so we are in the settings of the case 3 ).

Finally we conclude that if the time-optimal trajectory between two potential breakpoints $A$ and $B$ hits an obstacle then there is a potential breakpoint $C$ between $A$ and $B$ such that the time-optimal trajectory with obstacles from $A$ to $B$ passes $C$. 

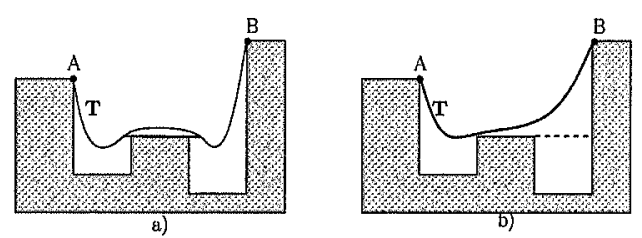

Fig. 14. Non-monotonic obstacle case, the obstacle is lower than $A$ and $B$

\section{Elimination theory}

In this appendix we briefly introduce some notions and facts of computational algebraic geometry and commutative algebra about polynomials of $n$ variables $x_{1}, \ldots, x_{n}$ (see [11]):

- The set of all polynomials at $x_{1}, \ldots, x_{n}$ with coefficients in the field $k$ is denoted $k\left[x_{1}, \ldots, x_{n}\right]$.

- The product $c x_{1}^{\alpha_{1}} \cdots x_{n}^{\alpha_{n}}, c \in k$, is called a leading term of the polynomial if $\sum_{i=1}^{n} \alpha_{i}$ is the biggest among all the sums of the powers.

- A subset $I \subseteq k\left[x_{1}, \ldots, x_{n}\right]$ is an ideal if it satisfies:

- $0 \in I$,

- if $f, g \in I$ then $f+g \in I$,

- if $f \in I$ and $h \in k\left[x_{1}, \ldots, x_{n}\right]$ then $h f \in I$.

- If $f_{1}, \ldots, f_{s} \in k\left[x_{1}, \ldots, x_{n}\right]$ then $\left\langle f_{1}, \ldots, f_{s}\right\rangle=\left\{\sum_{i=1}^{s} h_{i} f_{i} \mid h_{1}, \ldots, h_{s} \in k\left[x_{1}, \ldots, x_{n}\right]\right\}$ is an ideal of $k\left[x_{1}, \ldots, x_{n}\right]$.

- We can think of $\left\langle f_{1}, \ldots, f_{s}\right\rangle$ as consisting of all "polynomial consequences" of the equations $f_{1}=\ldots=f_{s}=0$.

- An ideal $I$ is finitely generated if there exist $f_{1}, \ldots, f_{s} \in k\left[x_{1}, \ldots, x_{n}\right]$ such that $I=\left\langle f_{1}, \ldots, f_{s},\right\rangle$. $f_{1}, \ldots, f_{s}$ are a basis of $I$.

- The lexicographical order lex is determined as follows: $x_{1}^{\alpha_{1}} \cdots x_{n}^{\alpha_{n}}>_{\text {lex }} x_{1}^{\beta_{1}} \cdots x_{n}^{\beta_{n}}$ if in the product $x_{1}^{\alpha_{1}-\beta_{1}} \cdots x_{n}^{\alpha_{n}-\beta_{n}}$ the leftmost nonzero entry among $\alpha_{i}-\beta_{i}$ is positive.

- The set $\left\{g_{1}, \ldots, g_{s}\right\} \subset I$ is called a Groebner basis of $I$ iff the leading term of every element of $I$ is divisible into the leading term of at least one $g_{i}$.

- The Groebner basis of any $\emptyset \neq I \subset k\left[x_{1}, \ldots, x_{n}\right]$ can be found in finite number of steps by Buchberger's algorithm.

- Given $I=\left\langle f_{1}, \ldots, f_{s}\right\rangle \subset k\left[x_{1}, \ldots, x_{n}\right]$ the $l$-th elimination ideal $I_{l}, l \leq 1$, is the ideal of $k\left[x_{l+1}, \ldots, x_{n}\right]$ defined by $I_{l}=I \cap k\left[x_{l+1}, \ldots, x_{n}\right]$. Thus, $I_{l}$ consists of all consequences of $f_{1}=\ldots=f_{s}=0$ which eliminate the variables $x_{1}, \ldots, x_{l}$.

- The Elimination Theorem: Let $I \subset k\left[x_{1}, \ldots, x_{n}\right]$ be an ideal and let $G$ be a Groebner basis of $I$ with respect to lex order $x_{1}>x_{2}>\ldots>x_{n}$. Then, for every $1 \leq l \leq n$, the set $G_{l}=G \cap k\left[x_{l+1}, \ldots, x_{n}\right]$ is a Groebner basis of the $l$-th elimination ideal $I_{l}$. 